\title{
The Resisting Mechanism during the Large Normal Deflection of Thin Shells Subjected to Follower Forces
}

\author{
by Thomas George*, Member Hiroo Okada*, Member \\ Nobuyoshi Fukuchi**, Member
}

\begin{abstract}
Summary
The load resistance mechanism of thin shells, such as the submerged shell structures in underwater marine constructions, are in principle of a nonconservative nature since the associated loads are the hydrostatic pressure and drag forces of the follower type.

The general governing equations of equilibrium for thin shells are available from the literature in various details. In this research we consider shells defined in a monoclinically convected coordinate system subjected to follower loads and undergoing large deformations. The feasibility and significance of the theoretical formulations have already been substantiated through various numerical simulation results.

In the simultaneous equations governing the equilibrium of shells, the presence of terms related to the shell curvature and other consequential terms such as the Christoffel symbols, clearly make them a substantially different class from the plate theory. This distinction, which may otherwise be denoted as the 'Form Effect' in more common terminology, renders the shell theory a lot more complicated and one that needs in-depth analytical effort to unravel the full significance of all the possible implications.

The present paper elaborates on the mechanism of large deformation by studying the share of different stiffness factors on the total load resistance equilibrium picture of shells from the very shallow to the deep curvature range going through the subsequently increasing loading stages. This study concentrates mainly on the equilibrium in the normal direction. The resisting mechanism due to the extensional and the bending stiffness parts are considered as the two main entities and their subdivisions and other separable factors are studied in unison to draw out an overall picture. The partial cylindrical and spherical shells are considered and the results bring out the clear distinction between resistance mechanism of the two shells, especially in the deep curvature region.

These results in their total details are believed to be helpful in understanding the deformation mechanism and formulate some design philosophy for shells and thereby serve as a guideline for appropriate formulations of the governing equations in particular cases.
\end{abstract}

\section{Introduction}

The importance of codification in the field of practical designs is accepted beyond doubt. Standards are necessary to implement an efficient and fast design procedure. Theoretical knowledge is brought to the practitioner through the analytical efforts and simplified interpretations adaptable to his needs. Structural design is generally based on the codes and standards, which makes it all the more important to update it with theoretical progress to meet the ever increasing demand for novel ventures.

In the field of marine structural systems, shell systems are projected to play an increasing role in the future

* College of Engineering, Osaka Prefecture University

** Faculty of Engineering, Kyushu University

Received 10th Jan. 1996

Read at the Spring meeting 15, 16th May 1996 demands for deep sea constructions. Considering the enormous structural strength and stability that can be achieved by effectively utilizing the 'Form Effect' due to curvature of the shells, the possibility of lighter and safer constructions in the ultra-high pressure environment of ocean depth could become possible. The present level of knowledge about shells go into various details in different heoretical and practical investigations ${ }^{122)}$, as a result of which the theoretical and subsequently the practical standards envelop a sphere of criteria which are derived from not so clear visualizations of the characteristics of each particular type of shell.

This research in its earlier stages has formulated a very detailed ${ }^{3)}$ general governing equation from the first principles, for the large deformations of shells subjected to follower loads. There the shells were defined in the monoclinically convected coordinate system and the deflected states were expressed using the metric and curvature tensors of the deformed state written in terms of the original metric and curvature tensors and the 
deflection values. Numerical results were presented to substantiate the feasibility and significance of that formulation.

In another part of the research, the dynamic stability of shells under disturbed equilibrium conditions were investigated in elaborate details ${ }^{4)}$ through an investigation of the frequencies of natural vibrations and the dynamic responses to small disturbances of shells at their subsequent equilibrium positions. Further, using the method of disturbed small motions, several new aspects have been brought into light and many important conclusions have been derived ${ }^{5) 6}$ on the dynamic stability mechanism of shells.

In the present paper, the equilibrating mechanism of the shell is studied using the governing equation for the normal direction as the basis, by analyzing the variations in the relative contributions from the groups of extensional and bending stiffness terms to the total resistance mechanism of two different types of shells. Further more, detailed analyses of different terms in the extensional and bending parts containing the principal strains and their cross components, the inplane deflections, the Christoffel symbols and the curvature tensors of mixed variance were carried out. This investigation helped to get some detailed knowledge of the resisting mechanism due to each of the above factors, both in the initial deflection and the large deflection stages.

It is generally understood that the mechanism of the plate transforms into that of the shell by the inclusion of curvature and its consequences. However, a clearcut statement of the facts in this field was generally not available in any easy to understand way. It is shown here that even the bending stiffness part that dominates the plate deflection mechanism is not fully sufficient in the large deflection stages. On the contrary, the extensional stiffness part can solely dictate the entire deflection mechanism of very deep shells. The curvature ranges at which the bending and the extensional parts share the strength scenario in varying proportions is presented here. Also, the significance and effective curvature zone of inplane deflection terms, the Christoffel symbols and the curvature tensors of mixed variance are illustrated. It shows that the shell as a whole is not possibly a candidate for classification or consideration as a single structural type, but differs in various details and each of the differing classes of shells has to be considered as a separate entity with particular characteristic governing equations.

From this analysis, it becomes possible to formulate the governing equation for the normal deflection of shells on a case by case basis depending on the curvature under consideration and thus save unnecessary efforts in numerical analysis with sufficient accuracy. However, it may be noted that this conclusion does not necessarily hold in the analysis of dynamic phenomena, where it requires to consider the nonlinearities due to some of the smaller effects also.

The results of the present research makes it possible to categorize the entire curvature range of shells into six different classes in general, from the plate to the very deep shell. As a result, the governing mechanism of internal resistance for each of these classes become visible to the practitioner and might help him to formulate some useful design codes in the future.

Partial cylindrical and spherical shells with curvatures ranging from very shallow to very deep were chosen as the representative cases of singly and doubly curved shells. The boundary conditions are assumed to be simply supported with the inplane deflections arrested along all the edges. The shell perimeter is considered to produce a projected square base of unit area, and the loading is assumed to be the uniformly distributed hydrostatic pressure. The Galerkin method is used for the numerical solution of the equilibrium problem. The present results are derived for the entire shell as an integral unit and not just for some particular points on the shell in a random selection. Thus, these results may be taken for their overall effect, which may be considered qualitatively true for any single point on the shell. Also, the post-critical stability range of equilibrium conditions are not considered here for it represents an entirely different class of mechanism altogether.

\section{Theoretical Formulations}

The derivation process of the general governing equations from the fundamentals of tensor continuum mechanics is not detailed here. The treatment in this section starts from the final form of the governing equations and thereby proceed to the details necessary for the purpose of this paper. However, background materials are explained to the necessary levels and the terminology is restated wherever found necessary.

\section{1 Coordinate Definition}

The shell continuum is defined here in the general convected coordinates. The system of monoclinically convected coordinates defined over the middle surface of a general shell is shown in Fig. 1. The figure also shows the geometry and dimensions assumed for the partial cylindrical and spherical shells used for numerical calculations in this paper.

The shell thickness $t$ is assumed to be uniform. By virtue of considering only thin shells, the KirchhoffLove hypotheses are assumed to be true during the entire finite deformation process.

\section{2 Equilibrium Equations}

The general governing equations for the finite deformations of thin shells are stated below. The range convention for all the Greek indices appearing here and elsewhere in this paper are to be taken as $\alpha, \beta, \gamma, \cdots=$ 1,2 .

$$
\begin{aligned}
& N_{(\mathrm{a})}^{\alpha \beta}\left\|_{(\mathrm{a})}+M^{\alpha \gamma}\right\|_{\alpha} B_{\gamma}^{\beta}=-p^{\beta}-m^{\alpha} B_{\alpha}^{\beta} \\
& N^{\alpha \beta} B_{\alpha \beta}-M_{(\mathrm{a})}^{\alpha \beta}\left\|_{\alpha \beta}=-p^{3}+m^{\alpha}\right\|_{(\mathrm{a})}{ }^{\alpha}
\end{aligned}
$$




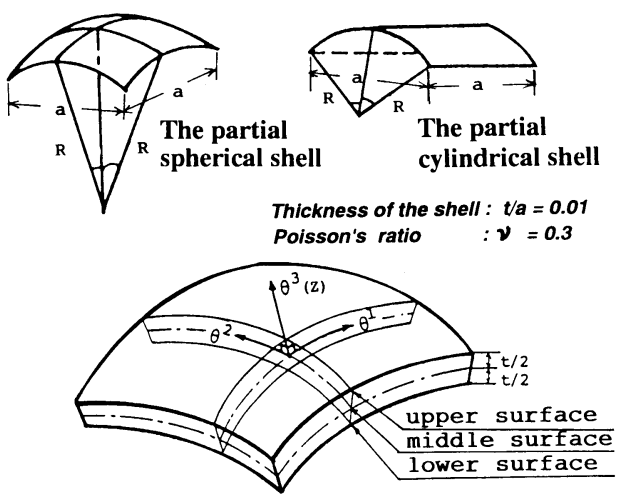

Fig. 1 The Definition of monoclinically convected coordinate axes and the geometry of two particular shell types

All the quantities in these equations represent the state of the middle surface after deformation. The symbol 'U' represents the 2-dimensional (surface) covariant differentiation of the preceding middle surface quantities with respect to the subscripts. The symbol (a)denotes that these differentiations are to be done over the quantities after deformation.

$N^{\alpha \beta}, M^{\alpha \beta}$ and $B_{\alpha \beta}$ are the membrane force tensor, the moment tensor and the curvature tensor of the middle surface after the deformation, respectively. The tangential and the normal component in the positive $z$ direction of the applied load are denoted respectively by $p^{\beta}$ and $p^{3}$, and $m^{\alpha}$ is the sum of the surface tractions and body forces contributed moment load. The loading is assumed to be the uniformly distributed follower type. It is necessary that the physical interpretations ${ }^{3}$ for the different tensor components are made, wherever necessary.

Now, the membrane force tensor, the moment tensor and the curvature tensor can be expressed in the following forms.

$$
\begin{gathered}
N^{\alpha \beta}=D a^{\alpha \beta \delta \lambda} \varepsilon_{\delta \lambda}+K a^{\epsilon \beta \eta \xi}\left(b_{\phi}^{\phi} \delta_{\varepsilon}^{\alpha} \delta_{\eta}^{\delta} \delta_{\xi}^{\lambda}-b_{\varepsilon}^{\alpha} \delta_{\eta}^{\delta} \delta_{\xi}^{\lambda}\right. \\
\left.\quad-b_{\xi}^{\lambda} \delta_{\varepsilon}^{\alpha} \delta_{\eta}^{\delta}\right) \kappa_{\delta \lambda} \\
M^{\alpha \beta=}=K a^{\alpha \beta \delta \lambda} \kappa_{\delta \lambda}+K a^{\varepsilon \beta \delta \lambda}\left(b_{\phi}^{\phi} \delta_{\varepsilon}^{\alpha}-b_{\varepsilon}^{\alpha}\right) \varepsilon_{\delta \lambda} \\
B_{\alpha \beta}=b_{\alpha \beta}+\kappa_{\alpha \beta}
\end{gathered}
$$

In the above equations, $D$ and $K$ are respectively the Extensional and the Bending stiffness parameters. $a^{\alpha \beta \gamma \delta}$ is the Elasticity tensor of the middle surface before deformation. The initial curvature tensor is given by $b_{\alpha \beta}$ whereas $b_{\alpha}^{\gamma}$ represents the curvature tensor of mixed variance. $\varepsilon_{\alpha \beta}$ is the strain tensor and $\kappa_{\alpha \beta}$ is the change of curvature tensor of the middle surface. The above quantities are defined as given below, where $E$ is the Young's Modulus of Elasticity, $\nu$ is the Poisson's ratio and $t$ is the shell thickness.

$$
\begin{aligned}
& D=\frac{E t}{\left(1-\nu^{2}\right)}, \quad K=\frac{E t^{3}}{12\left(1-\nu^{2}\right)} \\
& a^{\alpha \beta \gamma \delta}=\left(\frac{1-\nu}{2}\right)\left[a^{\alpha \gamma} a^{\beta \delta}+a^{\alpha \delta} a^{\beta \gamma}\right]+\nu a^{\alpha \beta} a^{\gamma \delta}
\end{aligned}
$$

$$
\begin{aligned}
\varepsilon_{\alpha \beta}= & \frac{1}{2}\left(A_{\alpha \beta}-a_{\alpha \beta}\right) \\
= & \frac{1}{2}\left(\left.u_{\alpha}\right|_{\beta}+\left.u_{\beta}\right|_{\alpha}+\left.\left.u^{i}\right|_{\alpha} u_{i}\right|_{\beta}\right) \\
= & \frac{1}{2}\left\{u_{\alpha, \beta}+u_{\beta, \alpha}-2\left(b_{\alpha \beta} u_{3}+\Gamma_{\alpha \beta}^{\lambda} u_{\lambda}\right)\right. \\
& +b_{\alpha}^{\lambda} b_{\lambda \beta}\left(u_{3}\right)^{2}+u_{3, \alpha} u_{3, \beta} \\
& +b_{\alpha}^{\lambda}\left[\left(b_{\beta}^{\rho} u_{\rho}+u_{3, \beta}\right) u_{\lambda}+\left(\Gamma_{\lambda \beta}^{\rho} u_{\rho}-u_{\lambda, \beta}\right) u_{3}\right] \\
& \left.+b_{\beta}^{\lambda}\left(u_{\lambda} u_{3, \alpha}-u_{\lambda, \alpha} u_{3}\right)\right\} \\
\kappa_{\alpha \beta}= & \left.u^{3}\right|_{\alpha \beta}-\left.\left.u^{3}\right|_{\gamma} u^{\gamma}\right|_{\alpha \beta} \\
& +\frac{1}{2}\left(\left.\left.u^{\gamma}\right|_{\gamma} u^{\delta}\right|_{\delta}-\left.\left.u^{\gamma}\right|_{\delta} u^{\delta}\right|_{\gamma}-\left.\left.a^{\gamma \delta} u^{i}\right|_{\gamma} u_{i}\right|_{\delta}\right) b_{\alpha \beta} \\
= & u_{3, \alpha \beta}-b_{\alpha}^{\lambda} b_{\beta \lambda} u_{3}-\Gamma_{\alpha \beta}^{\lambda} u_{3, \lambda}+\left(b_{\beta}^{\lambda} u_{3, \alpha}+b_{\alpha}^{\lambda} u_{3, \beta}\right) u_{3, \lambda} \\
& +\frac{1}{2}\left[b_{\lambda}^{\lambda} b_{\rho}^{\rho}\left(u_{3}\right)^{2}-b_{\rho}^{\lambda} b_{\lambda}^{\rho}\left(u_{3}\right)^{2}\right] b_{\alpha \beta}
\end{aligned}
$$

Here, $\alpha_{\alpha \beta}$ and $A_{\alpha \beta}$ are respectively the metric tensors of the middle surface before and after the deformation. $u^{i}$ or $u_{i}(i=1,2,3)$ represent the covariant and contravariant deflection components in the three coordinate directions. The vertical bar '|' symbol denotes the three dimensional (spacial) covariant differentiation with respect to the subscript. As usual, $\Gamma_{\alpha \beta}^{\lambda}$ are the Christoffel symbols, $\delta_{\alpha}^{\beta}$ are the Kronecker deltas and the comma notation is employed here to denote partial differentiations.

By substituting for the membrane force tensor, the moment tensor and the curvature tensor, the equilibrium equations of the shell can be formulated from Eq. (1) and Eq. (2). The expressions given here for these quantities were deduced after a strict order evaluation which retained only the most significant of the terms. The result of this substitution is not given here in terms of deflection components. However, a modified form in terms of the middle surface strain, the curvature tensor and the change of curvature tensor of the middle surface after deformation, is provided in the next section to facilitate further background for analysis.

Now, the deflection components $u_{k}(k=1,2,3)$ can be considered here in the form of an algebraic summation series, as follows:

$$
u_{k}=\sum_{i=1}^{m} \sum_{j=1}^{n} U_{k(i, j)} \phi_{k}\left(\theta^{1}, \theta^{2}\right)
$$

Here, $U_{k}$ represent the deflection coefficient and $\phi_{k}\left(\theta^{1}, \theta^{2}\right)$ may be considered as a double trigonometric function, where $\theta^{1}$ and $\theta^{2}$ are the angular coordinates of the middle surface in the principal directions.

\section{3 The Mechanism of Normal Deformations}

This paper considers the mechanism during the normal deformations of the shell. Thus, Eq. (2) which is the governing equation in the normal direction is to be analyzed for bringing out its characteristics. For the sake of convenience, Eq. (2) is represented in the following form where the $\mathcal{E}$ term denotes the group of terms that club together with the Extensional stiffness parameter $D$ and $B$ term denotes the group of terms that club together with the Bending stiffness parameter $K$.

$$
\mathcal{E}+\mathscr{B}=-p^{3}
$$


the following components, where the $\mathbf{E}$ terms and the $\mathbf{B}$ terms denote a division based on the characteristics of each group of terms.

$$
\left.\begin{array}{l}
\mathcal{E}=\mathbf{E} 1+\mathbf{E} 2+\mathbf{E} 3+\mathbf{E} 4 \\
\mathcal{B}=\mathbf{B} 1+\mathbf{B} 2+\mathbf{B} 3+\mathbf{B} 4
\end{array}\right\}
$$

The significance and meaning of this arbitrary subdivision is visible when each group of terms is studied from the following detailed representation of their structures. Here again, for the sake of convenience and easy visualization effect, the equation is given in terms of strain components, curvatures and the change of curvature.

$$
\left.\begin{array}{rl}
\mathbf{E} 1= & D\left(a^{11}\right)^{2} \varepsilon_{11} B_{11} \\
\mathbf{E} 2= & D \nu a^{11} a^{22}\left[\varepsilon_{11} B_{22}+\varepsilon_{22} B_{11}\right] \\
\mathbf{E} 3= & D\left(\frac{1-\nu}{2}\right) a^{11} a^{22}\left[\left(\varepsilon_{12}+\varepsilon_{21}\right)\left(B_{12}+B_{21}\right)\right] \\
\mathbf{E} 4= & D\left(a^{22}\right)^{2} \varepsilon_{22} B_{22} \\
\mathbf{B 1}= & K\left(a^{11}\right)^{2}\left[\kappa_{11} B_{11}\left(b_{2}^{2}-b_{1}^{1}\right)-\kappa_{11,11}-\varepsilon_{11,11} b_{2}^{2}\right] \\
\mathbf{B} 2= & -K \nu a^{11} a^{22}\left[\kappa_{11,22}+\kappa_{22,11}+\varepsilon_{11,22} b_{1}^{1}+\varepsilon_{22,11} b_{2}^{2}\right] \\
\mathbf{B} 3= & -K\left(\frac{1-\nu}{2}\right) a^{11} a^{22}\left[2\left(\kappa_{12,12}+\kappa_{21,12}\right)\right. \\
& \left.+\left(\varepsilon_{12,12}+\varepsilon_{21,12}\right)\left(b_{1}^{1}+b_{2}^{2}\right)\right] \\
\mathbf{B 4}= & K\left(a^{22}\right)^{2}\left[\kappa_{22} B_{22}\left(b_{1}^{1}-b_{2}^{2}\right)-\kappa_{22,22}-\varepsilon_{22,22} b_{1}^{1}\right]
\end{array}\right\}
$$

First of all, the terms $\mathbf{E} 1$ and $\mathbf{B} 1$ contain terms grouped together with the metric tensor in the first principal direction $\theta^{1} \quad$ (the term 'primary direction' will be used in further discourse) and the terms $\mathbf{E} 4$ and $\mathbf{B} 4$ contain terms grouped together with the metric tensor in the second principal direction $\theta^{2}$ (the term 'secondary direction' will be used in further discourse). The terms $\mathbf{E} 1$ and $\mathbf{E} 4$ represent the stretch in the respective principal directions, whereas the terms $\mathbf{B} \mathbf{1}$ and $\mathbf{B} 4$ represent the bending in the respective principal directions.

Next, the terms E2, E3, B2 and B3 are groups of terms accompanying the product of metric tensors in the principal directions and characterized by the presence of the Poisson effect. They are basically the cross components of stretching and bending in the principal directions. The difference lies in the fact that the terms E2 and $\mathbf{B} 2$ represent the effect due to strains and curvatures or its changes in the principal directions, whereas the terms $\mathbf{E} 3$ and $\mathbf{B 3}$ are associated with the twist components of the same.

The terms $\mathcal{E}$ and $\mathcal{B}$ will be denoted in further discourse as the Extensional part and the Bending part, respectively. Also, their subdivisions will be denoted as the Component terms. The above subdivision is to serve as the basis for all the evaluations made of the resistance mechanism of shells during the numerical analyses conducted in the remaining part of this paper.

\section{Numerical Analysis of the Resistance Mechanism}

In shell theory, which may be broadly divided into shallow shells and deep shells based on the curvatures, several classification schemes are available. In the design of shells however, the classifications cannot be of any value until they are easily visualizable. In this paper, an attempt is made to classify the shells in terms of the common terminology that does not need any expertise in the intricacies of the shell theory to understand.

The shell type that should be analyzed, the boundary conditions to be selected and the type of loading that requires attention do exist in several permutations. Here, the partial cylindrical shells and the partial spherical shells are selected as the most prominant representatives of the singly and doubly curved shells, respectively. The boundaries are considered simply supported, with all the inplane deflections arrested along the edges. The loading is considered to be the uniformly distributed hydrostatic pressure acting on the entire shell surface in the anti-radial direction.

The shell geometry is considered to be that which produces a projected square base of unit area. The principal chord lengths $\left[a=l_{1}=l_{2}, t / a=0.01\right]$ are unity by virtue of the choice of projected unit square base. The shell thickness $t$ is considered uniform and sufficiently small enough in comparison with the radius $R$, to satisfy the fundamental assumptions. The numerical value of $E=1.96 \times 10^{11} \mathrm{~N} / \mathrm{m}^{2}$ for the Young's modulus and the Poisson's ratio of $\nu=0.3$ are used for numerical calculations.

The differential geometry of a toroidal shell definition is used to express the basic values of the shell middle surface. The corresponding equations for the metric and curvature tensors and the Christoffel symbols can easily be formulated from first principles ${ }^{3)}$. The 'primary direction' (see sec. 2.3) of partial cylindrical shells is chosen in its longitudinal direction.

For numerical analysis, the equilibrium equations [Eqs. (1),(2)] under static considerations are solved using the Galerkin method. Here, 16 modal combinations $[m, n=4$ in Eq. (10)] are used. Curvature ranges of shells were selected from the plate (Radius, $R / a=\infty$ ) to the subtended angle of $\pi$ radians at the center (Radius, $R / a=0.5)$ for the deep shell. As a result, about 40 different curvature values each for the partial cylindrical and spherical shells were used for this analysis.

In the numerical analysis of Eq. (11) to determine the resistance mechanism of shells, instead of considering only a single monitoring point, for example the center of the shell, the average value of several symmetric mesh points is calculated. However, the allowable error in satisfying Eq. (11) is restricted well within $1.0 \%$ by adjusting the number of mesh points.

\section{1 An Overview of Large Deformation and the} Initial Resistance Mechanism

The numerical calculation of large deformation of shells is performed using Eqs. (1) and (2) where the center of the shell is usually chosen as the monitoring point. In this paper, in addition to the center deflection, the average deflection also is calculated. The term average deflection denotes the average of analytically integrat- 


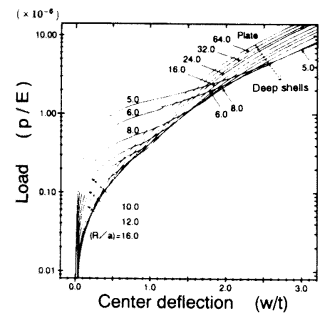

$(\mathrm{a}-1)$ Radius $(\mathrm{R} / \mathrm{a})=\infty \sim 5.0$

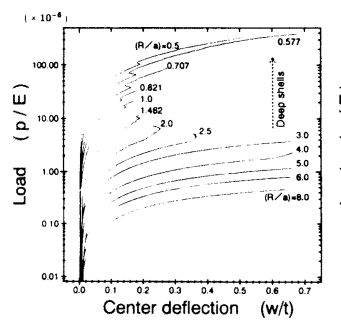

(a-2) Radius $(\mathrm{R} / \mathrm{a})=8.0 \sim 0.5$
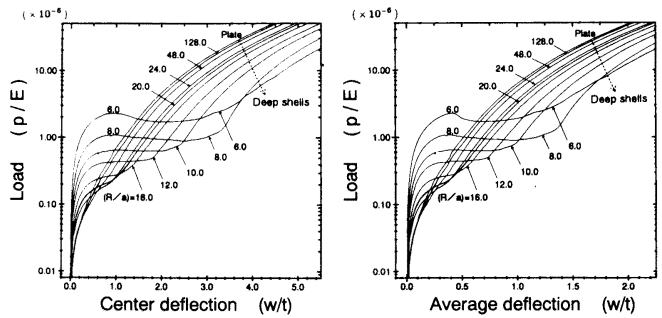

(b-1) Radius $(\mathrm{R} / \mathrm{a})=\infty \sim 6.0$

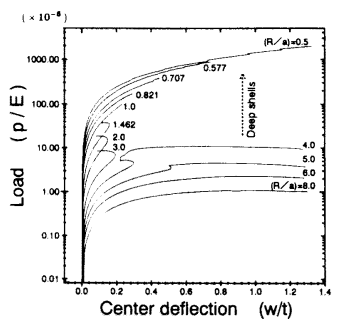

(b-2) Radius

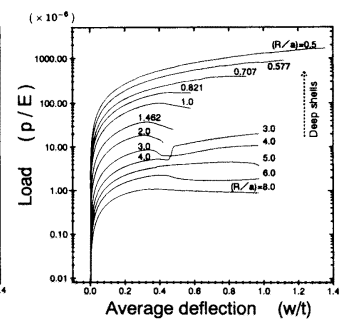

$(R / a)=8.0 \sim 0.5$

(b) Partial spherical shells

Fig. 2 Load-deflection curves of different shells

ing the deflection values $u^{3}$ over the whole shell surface using Eq. (10) and the calculated deflection coefficients. Normal deflections will be denoted hereafter using $w$ where $\mathrm{w}=u^{3}$ and the nondimensional value $\mathrm{w} / t$ will be used in the graphs.

In Fig. 2 the load-deflection curves of several shells are illustrated. Partial cylindrical and spherical shells are given side by side and the top row of graphs show the cases from that of the plate to the 'medium deep' shells. The bottom row of graphs show the cases from that of the 'medium deep' to 'very deep' shells. The terminology 'medium deep' and 'very deep' will be substantiated later in this paper. In each of these cases, the centre deflection graphs and the average deflection graphs are shown side by side for the sake of facilitating direct comparison.

First of all, by scanning through the graphs from the plate to the very deep shell, one can notice the gradual changes in the deflection pattern. For the plate, the well known case of normal bending deflection is very characteristic but for deeper shells the pattern undergoes drastic changes in several stages.

By comparing the center deflection and average deflection in each case, the similarities in pattern are very striking except that the numerical values of average deflections are smaller than the corresponding center deflections, which is a consequence of averaging over the whole shell. Also, the average deflections generally follow the forward deflection pattern even when the centre deflections are back folding (local reverse buckling). Thus, it may be concluded that by averaging we obtain a qualitatively representative picture for the whole shell. This is the reason why the following results on the calculation of the resistance mechanism of shells using Eq. (11) have been averaged for the whole shell over an appropriate number of mesh points.

The share of extensional and bending stiffness parts of the equilibrium equation for deformations in the normal direction has been determined in an earlier study $^{3)}$ using the corresponding principal diagonal coefficient of the stiffness matrix at the initial deflection stage. The qualitative idea that was proposed in that study is verified in the present study in a quantitative way by considering the whole of the equilibrium equation in an exact calculation. Fig. 3 shows that result where the influence parameter indicates the fraction of influence shared by a part.

For shallow shells shown towards the right of the figure, the bending part $(\mathcal{B})$ dominates the resistance mechanism almost completely. However, for deep shells at the left extreme the extensional part $(\mathcal{E})$ dominates the scenario. In between these two extremes, the shares of both $\mathcal{B}$ and $\mathcal{E}$ are present in different proportions.

Also, the curves for partial spherical shells are more shifted towards the shallow shell end than the partial cylindrical shell. This is very much according to the logical arguement that for doubly curved shells the effect of curvature appears at more shallow curvatures than singly curved shells for which the effect of bending in the primary direction lasts until deeper shells.

This total picture of initial effects due to the extensional and the bending parts can be analyzed in more logical details in Fig. 4 and Fig. 5 where the share of each component term is illustrated. Fig. 4 shows the 


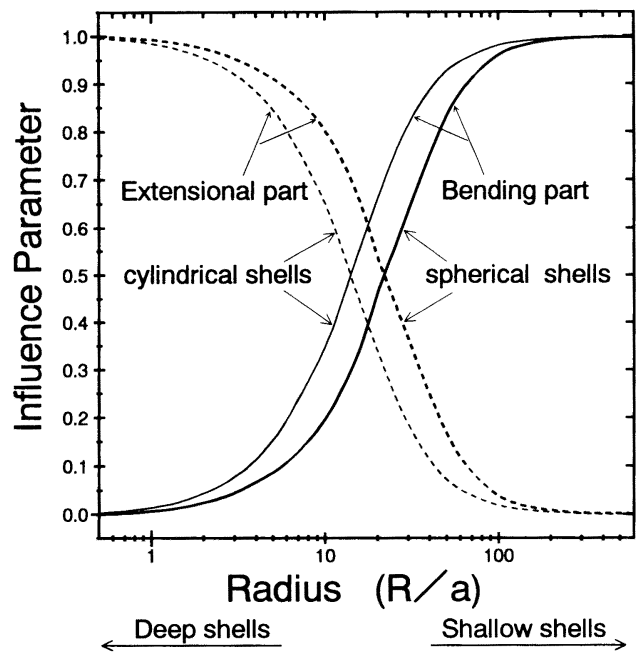

Fig. 3 The share of extensional and bending stiffness parts in the initial resistance mechanism of different shells

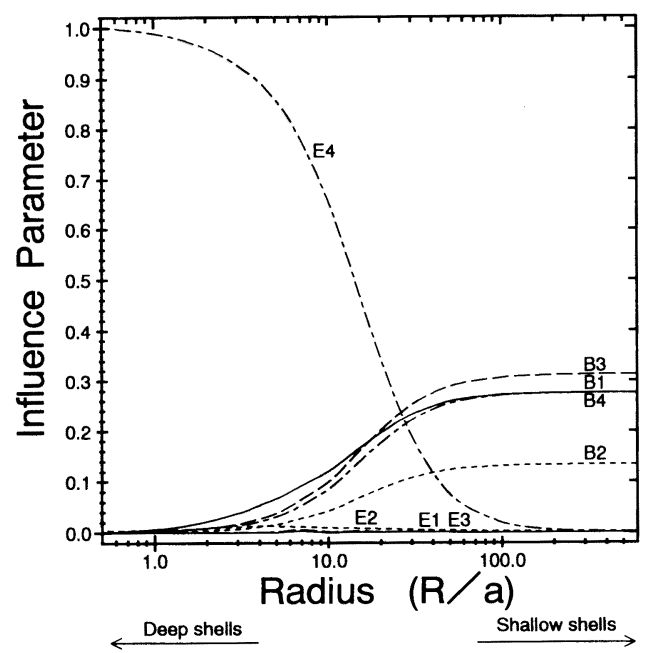

Fig. 4 The share of different components of the extensional and the bending stiffness parts in the initial resistance mechanism of partial cylindrical shells of different radii

case of partial cylindrical shells for which the shallow shell end is dominated in varying proportions by the bending components $\mathbf{B} 1$ to $\mathbf{B} 4$. The effect of principal bending components $\mathbf{B} \mathbf{1}$ and $\mathbf{B} \mathbf{4}$ are almost equal at this stage, as should be expected, and their sum having the largest influence. On the contrary, at the deep shell side the principal extensional part in the secondary (radial) direction E4 dominates an almost complete influence.

Comparing Fig. 3 and Fig. 4 brings out the interesting fact that for deep cylindrical shells the extensional component in the radial direction is the sole contributor

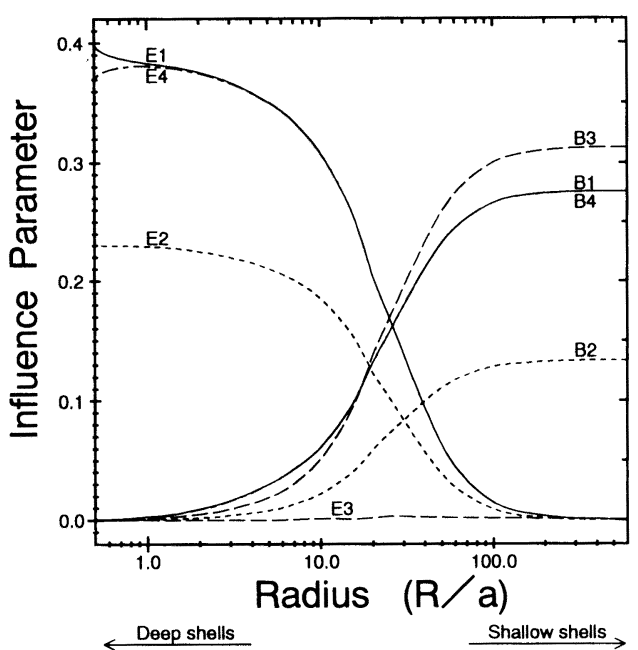

Fig. 5 The share of different components of the extensional and the bending stiffness parts in the initial resistance mechanism of partial spherical shells of different radii

to its resisting mechanism. This picture is different for deep spherical shells, as can be seen from Fig. 5 where the two principal extensional components E1 and E4 sharing a dominating influence at the deep shell end, along with the secondary influence of $\mathbf{E} 2$ component. In the case of both shells, the effect of inplane twist component $\mathbf{E} 3$ can be seen to be marginal for the whole range of curvatures.

The above results on the initial influence of various components are almost representative of the deformed equilibriums also. However, the mechanism of deformation is accompanied by geometrical changes which initiate some considerable changes in the resistance mechanism of the shell. This is studied in the following sections.

3.2 Resistance Mechanism in the Deformed States In studying the initial resistance mechanism, the fundamentals of the same have been clearly demonstrated. Now, the deformed states of a shell could give a slightly different scenario of resistance mechanism. Fig. 6 and Fig. 7 show respectively the cases of partial cylindrical shells and partial spherical shells.

The influence zones are considered as the range of influence parameters greater than $0.01 \quad(1.0 \%$ share of the total influence or more). The 'Threshold of static instability' is determined based on the combined estimation using the results of an earliar study ${ }^{4)}$ on the natural frequencies of vibration of the deflected shells and the ultimate loading values from the present study. The resistance mechanism is calculated for loading stages within this threshold load only, since the mechanism undergoes many changes beyond this point.

As for the method of reading these graphs, the bound 


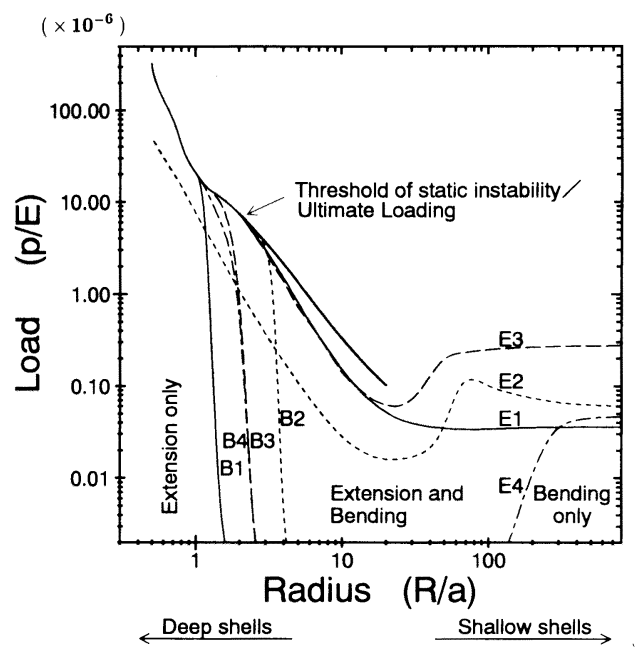

Fig. 6 Influence zones of different components of the extensional and the bending stiffness parts in the resistance mechanism of different partial cylindrical shells in the statically stable region

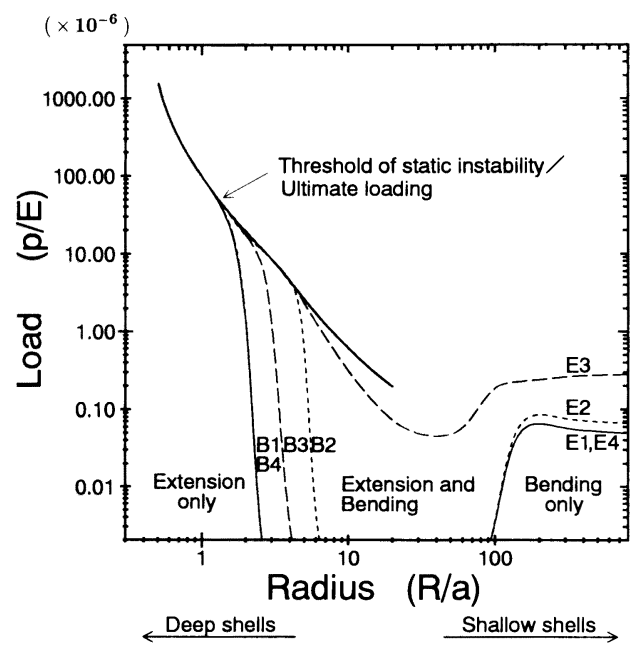

Fig. 7 Influence zones of different components of the extensional and the bending stiffness parts in the resistance mechanism of different partial spherical shells in the statically stable region

aries of influence zones for each component plotted here represents the appearance of that component when moving upwards through the direction of increasing load for each radius. A curve due to a component that does not appear at any loading stage can be considered to have its influence zone for all loading levels at that radius. Thus, at any radius the portion of an ordinate below the point of an intersection with a curve is the 'no influence zone' of load levels for that component.

In both figures, shallow shells towards the right hand corner are dominated by bending terms $\mathcal{B}$ only, until a higher loading stage where the influence of the extensional part $\mathcal{E}$ appears. This can be understood easily by considering that the deflected shell attains curvatures which bring on the extensional effect. Now, for deep shells towards the left end the picture is considerably different for cylindrical and spherical shells.

For the partial cylindrical shells shown in Fig. 6 the influence zones due to extensional components E1, E2 and E3 appear only at very high loading stages at or near the static instability. The initial influence zone at the deep shell end is completely dominated by the principal stretch component $\mathbf{E 4}$ in the radial direction. However, for the partial spherical shells shown in Fig. 7 the only extensional component that does not appear during the lower loading stages is the inplane twist E3 for the complete range of curvatures. As can be expected, the principal stretch components $\mathbf{E} 1$ and $\mathbf{E 4}$ are symmetric for all curvatures.

The appearance of influence zones due to the bending components $\mathbf{B} 1$ to $\mathbf{B} 4$ in the case of both the shells can be seen starting after the 'Extension only' zone and remains for the whole range of shallower shells towards the right of the figure. In the case of cylindrical shells in Fig. 6 the principal bending component $\mathbf{B 1}$ due to the primary direction appears first and the other components follow suit at shallower curvatures, as is expected.

The influence zone that appears in between the extremes is shared by both the extensional and the bending parts. The overall width of this zone is slightly larger for cylindrical shells due to the early appearance of the effect of bending at the deep shell side and the late disappearance of the effect of extension in the radial direction at the shallow shell end.

In Fig. 6 and Fig. 7 the range of curvatures for both the shells can be broadly divided into three zones, as shown. A representative curvature value from each of these zones is selected for some detailed illustrations in Fig. 8 and Fig. 9 where the top row shows the aggregate influence and the bottom row shows the influence due to the component terms of the bending and the extensional parts.

The sample shell of radius $R / a=128.0$ is selected from the 'Bending only' zone for both the shells. Figures $(\mathbf{a}-\mathbf{1})$ and $(\mathbf{b}-\mathbf{1})$ can be found to be of the same type for both shells except for the effect of unsymmetry of principal directions in the case of the cylindrical shell. It should be noted that the domination of the bending components disappear at higher loading stages, which is a result not directly visible from Fig. 6 in both qualitative and quantitative terms. This shows the importance of extensional terms for shallow shells also when considering large deformations.

Radius $R / a=16.0$ is selected from the 'Extension and Bending' zone for both the shells as shown in Figures $(\mathbf{a}-2)$ and(b-2). In this case the patterns do not match 


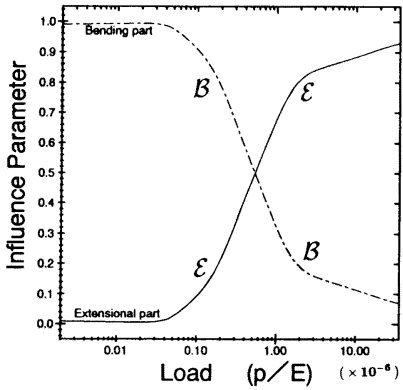

$(\mathrm{a}-1)$ Radius $(\mathrm{R} / \mathrm{a})=128.0$

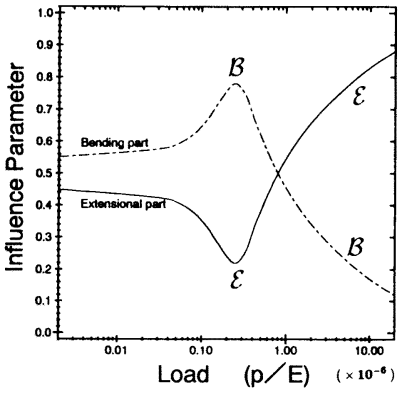

(a-2) Radius $(R / a)=16.0$

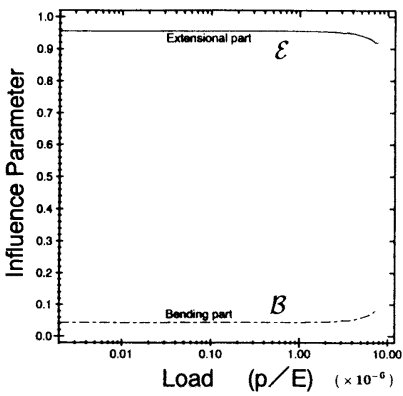

(a-3) Radius $(\mathrm{R} / \mathrm{a})=2.0$

\section{(a) Aggregate influence}

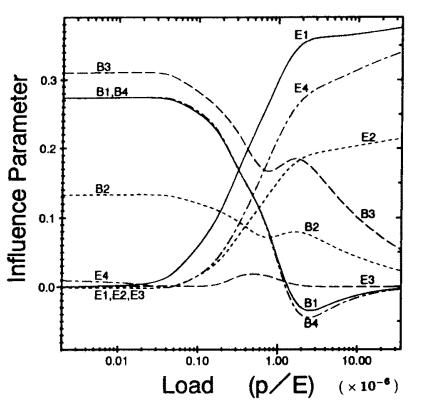

(b-1) Radius $(\mathrm{R} / \mathrm{a})=128.0$

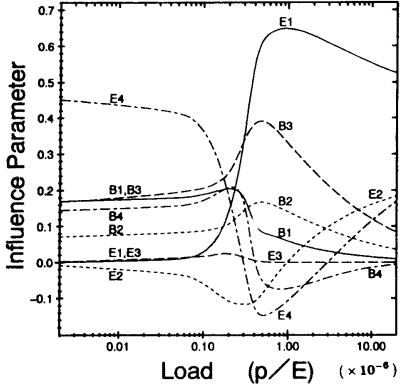

(b-2) Radius $(\mathrm{R} / \mathrm{a})=16.0$

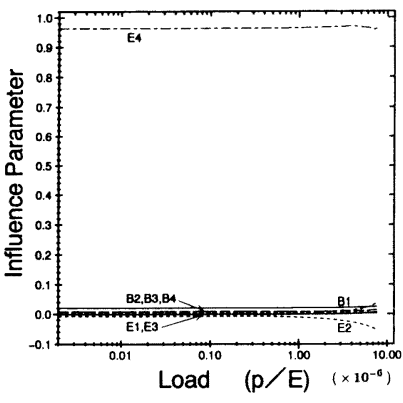

(b-3) Radius $(\mathrm{R} / \mathrm{a})=2.0$

\section{(b) Influence of component terms}

Fig. 8 Variations in the share of influence of different components of the extensional and the bending stiffness parts in the resistance mechanism of some partial cylindrical shells

between the two shells. For cylindrical shells in Fig. 8 the initial domination is by the bending part for the presence of its primary direction, which is the other way in Fig. 9 for the spherical shells. However, at higher loading stages the dominant parts reverse for the cylindrical shell while the initially dominant part of the spherical shell regains the position after an intermediate reversal of roles. The details of this picture given in (b-2) for both shells in terms of the corresponding components show the extemely complicated influence changes occuring around the static instability point of each shell.

The representative radius $R / a=2.0$ selected from the 'Extension only' zone for both the shells shown in Figures $(\mathbf{a}-\mathbf{3})$ and $(\mathbf{b}-\mathbf{3})$ are much less complicated. In the case of both the shells, the extensional part clearly dominates and its components follow the conclusions thus far.

It may be concluded from the above illustrations that for shells in the 'Bending only' zone, the extensional part dominates at higher loading stages. For shells in the 'Extension and Bending' zone, both the extensional and bending parts demonstrate a very complicated pattern of role playing. And the deep shells in the 'Extension only' zone follow the straight-forward pattern just as evident from the name of the zone. The above conclusions apply to both types of shells, in general.

\section{3 The Share of Influence of Smaller Factors}

The influences due to the extensional and the bending parts were studied in detail in the previous sections and the overall conclusions drawn thereby holds. However, the component parts are composed of several smaller terms that constitute each term of the equation. It might be worthwhile to study some of those terms and determine their share in the total picture. This process might further the aim of understanding the resistance mechanism to finer details.

Here we selected the inplane deflection components $u_{\alpha}\left(\right.$ or $\left.u^{\alpha}\right)$ or their derivatives, the Christoffel symbols $\Gamma_{\alpha \beta}^{\gamma}$ and the curvature of mixed variance $b_{\alpha}^{\beta}$ to study the influences of terms containing them in the extensional and the bending parts. Here again, the influence zones are considered as the range of influence parameters greater than $0.01(1.0 \%$ share of the total influence or more).

In Fig. 10 the influence of terms containing the inplane deflection components is illustrated. The initial influence only is shown in (a) for both the shells where 


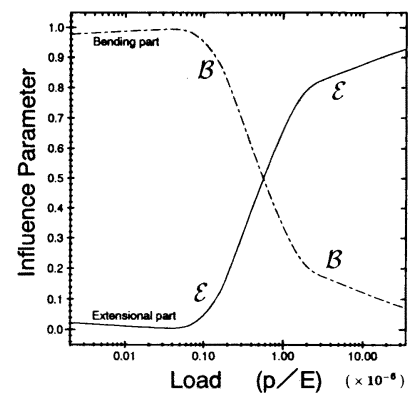

(a-1) Radius

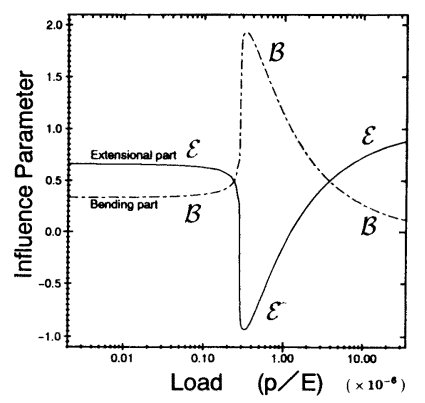

$(R / a)=16.0$

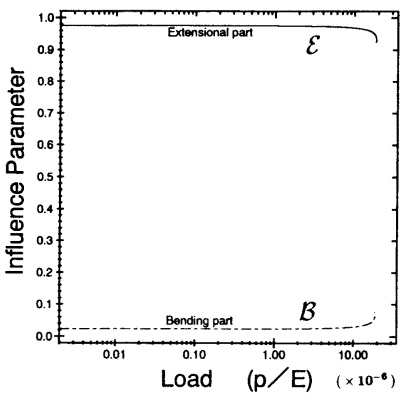

(a-3) Radius $(\mathrm{R} / \mathrm{a})=2.0$

\section{(a) Aggregate influence}

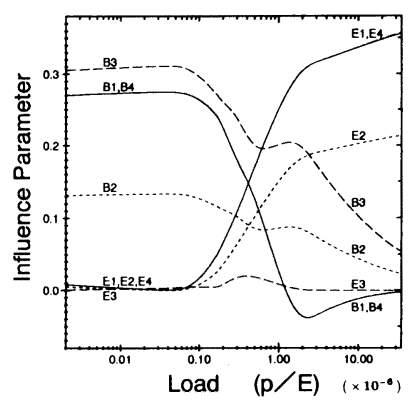

$(\mathrm{b}-1)$ Radius $(\mathrm{R} / \mathrm{a})=128.0$

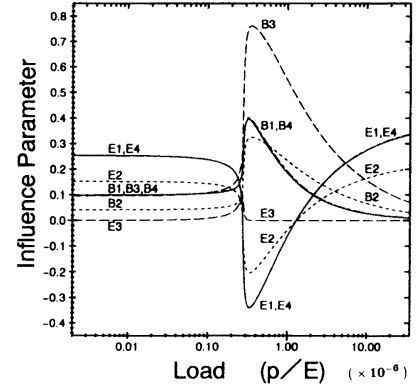

(b-2) Radius $(\mathrm{R} / \mathrm{a})=16.0$

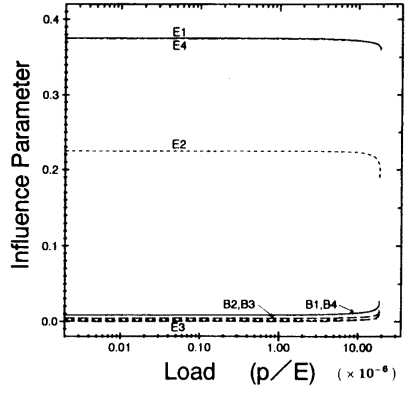

(b-3) Radius $(R / a)=2.0$

\section{(b) Influence of component terms}

Fig. 9 Variations in the share of influence of different components of the extensional and the bending stiffness parts in the resistance mechanism of some partial spherical shells

a maximum influence of up to $9.0 \%$ is found for shells in the medium and deep curvature zones. However, for shallow shells the influence is very small and for very deep shells the influence diminishes. It might be noted that for spherical shells the influence exists for a slightly wider range of curvatures than the cylindrical shells.

Although the initial influence is found to be very small for shallow curvatures, (b) shows the influence slowly picks up at higher loading levels, which has an earlier impact on partial spherical shells. The 'No influence zone' indicates the curvature range from the plate to shallow shells that has a less than $1.0 \%$ influence from the inplane deflection terms. These graphs show that the inplane deflection terms can not be neglected altogether, even for shallow shells, when considering large deformations. However, the same may be neglected in the bending part $\mathcal{B}$ since its significance lies only in the extensional part $\mathcal{E}$ for both types of shells.

The share of initial influence of the Christoffel symbols and their 'No influence zone' are shown in Fig. 11 for the partial spherical shells, since the partial cylindrical shells are found to be little influenced at all curvatures and loading stages. This curve shows that the 'No influence zone' extends from the curvature range of plates to deep shells, for which the maximum influence may exceed $6.0 \%$ at very deep shells. Here again the significance lies only with the extensional part $\mathcal{E}$ and the initial influence shown here is found to be true and applicable for all higher loading stages as well.

Quite contrary to the initial influence exhibited by the inplane deflection terms and the Christoffel symbols, the curvature tensors of mixed variance $\left(b_{\alpha}^{\beta}\right)$ were found to have little initial influence for all curvature ranges. However, at higher loading stages of deep shells the influence appears for both types of shells. This is shown in Fig. 12 where the 'No influence zone' extends from the plate to deep shells, which again is significant only in the extensional part $\mathcal{E}$. It was found that this influence becomes stronger near to the instabilities at large deformations, which makes them significant for deep shells.

The above illustrations show that the influences of all types of terms considered here are existing only in the extensional part. As a result, it may be concluded that the inplane deflection terms, the Christoffel symbols and the mixed variance of curvature terms may well be neglected in the bending part $\beta$ for a static analyis. As mentioned earliar, this conclusion does not necessarily hold for a dynamic analysis, since some of the above 


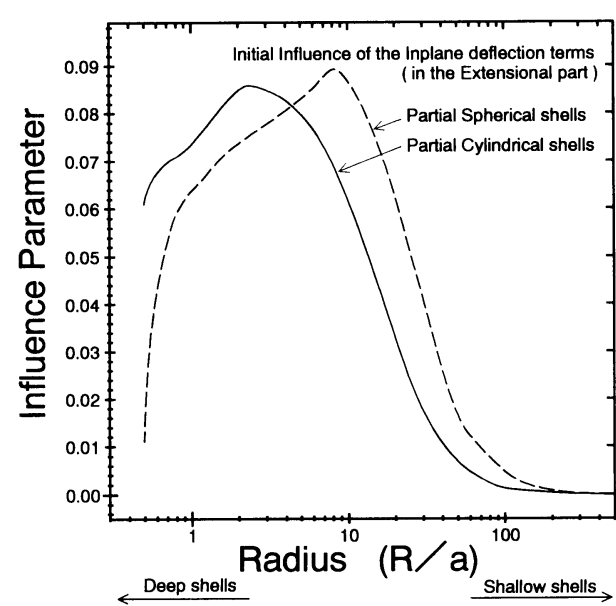

(a) Initial influence

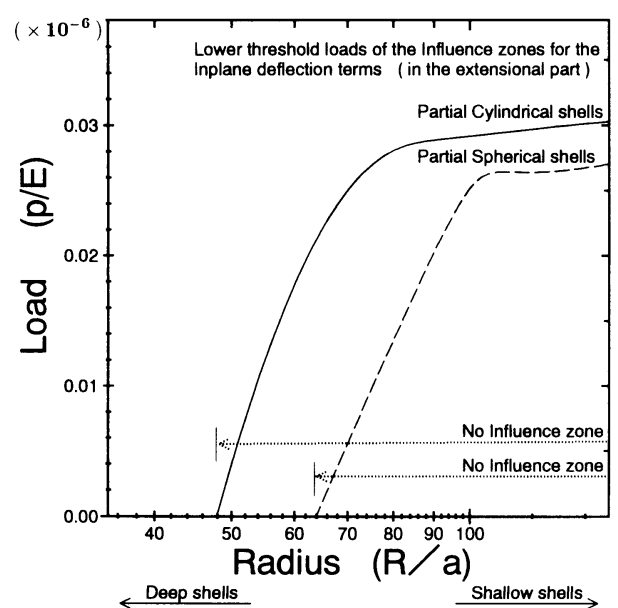

(b) Influence zones

Fig. 10 The share of initial influence and the lower threshold loads of influence zones of the inplane deflection terms in the resistance mechanism of different shells

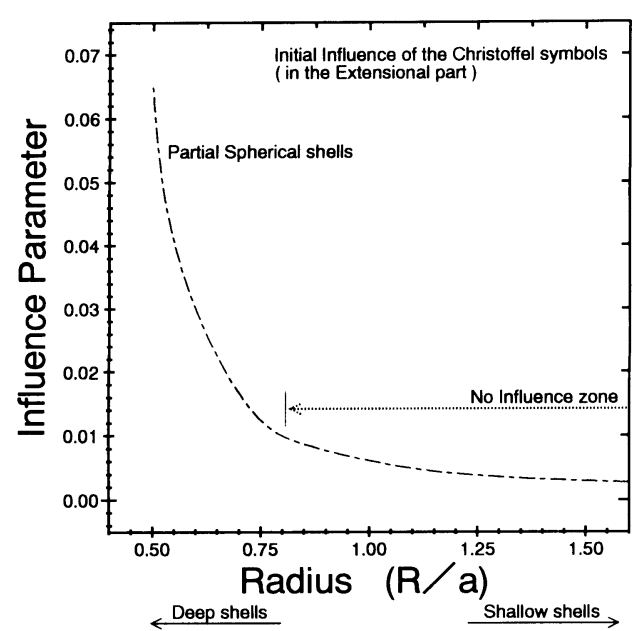

Fig. 11 The share of initial influence and the influence zone of the Christoffel symbols in the resistance mechanism of partial spherical shells

terms exhibit strong nonlinearities.

\section{4 A Categorization of Shell Curvatures}

The mechanism of resistance during the deformătion of shells was analyzed for two different shell types in the previous sections. The results obtained for the influences of several factors that constitute the total resistance mechanism have brought to light the interesting and complicated nature of realignments that occur between the bending and extensional stiffness terms during the deflection history of some shells. It is considered worthwhile to think of a meaningful categorization of shells based on these results.

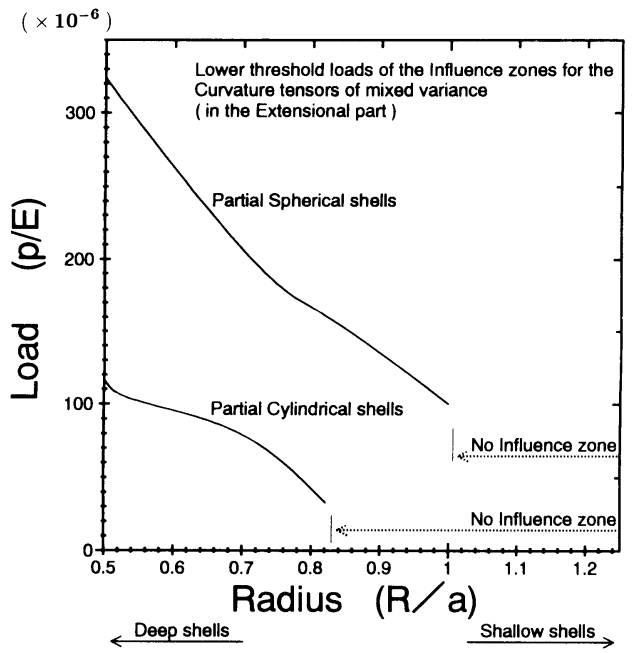

Fig. 12 The lower threshold loads of influence zones of the curvature tensors of mixed variance in the resistance mechanism of different shells

In Fig. 13 an illustration of the results of an attempt to categorize shells according to the differences in their deflection mechanisms is shown. It can be seen that there are some differences between the partial cylindrical and partial spherical shells. Generally, both the shell types have an 'extension only' zone, a 'bending only' zone and a 'combined influence' zone with respect to the initial influences of each term, as shown within the boxed area. The initial influence range of extensional terms extends more towards the shallow range for partial spherical shells than for partial cylindrical shells. On the contrary, the initial influence range of 


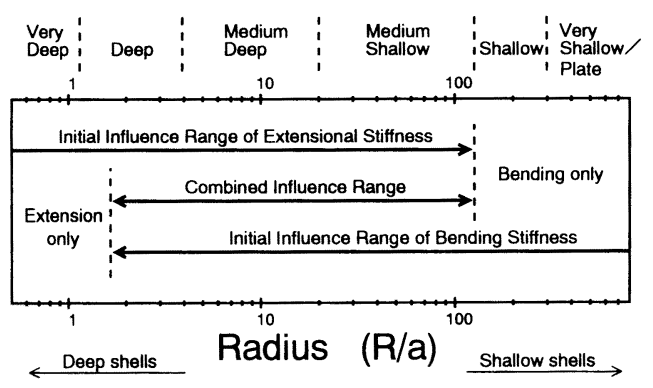

(a) Partial cylindrical shells

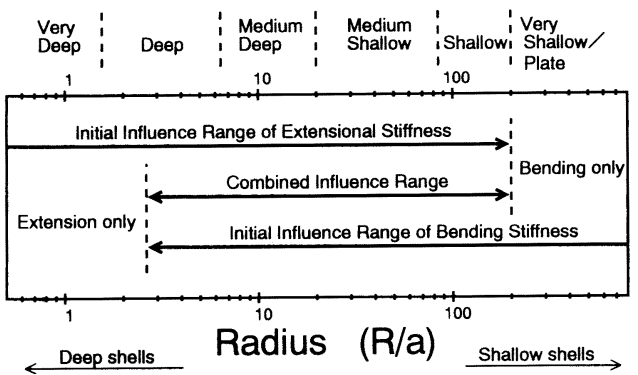

(b) Partial spherical shells

Fig. 13 A depiction of the initial influence regions of the extensional and the bending stiffness parts and an overall categorization of shell curvatures based on the different combinations of resistance mechanisms

bending terms extends more over to the deeper shells for partial cylindrical shells than for partial spherical shells. The mechanism of these differences are easily deducible from the results obtained so far.

Just above the boxed area is shown a subdivision of shell curvatures into six major types. The philosophy behind these subdivisions are drawn from all the previous results with an aggregrate consideration of the resistance and deformation mechanism of each shell type.

First of all, the 'very deep' shell denotes that range of curvatures at which the influence of bending terms are absent until very large loading stages and, the influences due to terms like the Christoffel symbols and the curvature tensor of mixed variance are present in the extensional part. In this range of curvatures, the resistance mechanism is purely extensional for all practical purposes.

On the other hand, within the 'very shallow/plate' range the shell behaves more like the plate or departs very slightly from it. Here, the shell is of the pure bending type until the large deformation stage, where the extensional part gains significance and remains so thereafter.

The 'deep' and the 'shallow' shells belong to those curvature ranges were the behaviour of 'very deep' and 'very shallow' shells begin to depart slightly from their initial behaviour, respectively. In the case of 'deep' shells, the influence of bending terms begin to appear at all the higher loading levels. similarly, the 'shallow' shells have some shared influence of the extensional part in the deformed states of equilibrium. In this range of curvatures, the shell shows a certain amount of complicated strength mechanism at higher loading levels.

Finally, the 'medium deep' and the 'medium shallow' -ranges of curvatures are by far the most intricate of all other ranges. Here, the shell shares the initial influence of both the extensional and the bending parts in varying proportions. For 'medium deep' shells there exist a clear point of 'static instability', which exists only vaguely for 'medium shallow' shells. Influence curves of the extensional component $\mathbf{E} 3$ for both types of shells and the influence curves of components E1 and E2 for partial cylindrical shells exhibit inflections within this range of curvatures. Also, the influence of inplane deflection terms are very high within this range. Numerical calculations on shells within the medium deep curvature range can reflect the effects of this complicated strength mechanism.

The above categorization can serve as a guideline or a starting point for investigating the shell problem in its various details. As for a simple suggestion into the rearragements that can be made in the general governing equation in the normal direction, given below is a selection of the most significant terms that should be considered when analyzing shallow shells. The terminology has already been explained before. The expressions for the membrane force and the bending moment may be considered as follows.

$$
\left.\begin{array}{l}
N^{\alpha \beta}=D a^{\alpha \beta \delta \lambda} \varepsilon_{\delta \lambda} \\
M^{\alpha \beta}=K a^{\alpha \beta \delta \lambda} \kappa_{\delta \lambda}
\end{array}\right\}
$$

From all the conclusions made so far, some drastic reductions may be made in the expressions for the strain tensor, and the change of curvature tensor.

$$
\left.\begin{array}{l}
\varepsilon_{\alpha \beta}=\frac{1}{2}\left\{-2 b_{\alpha \beta} u_{3}+u_{3, \alpha} u_{3, \beta}\right\} \\
\kappa_{\alpha \beta}=u_{3, \alpha \beta}
\end{array}\right\}
$$

The above situation applies only to shallow shells or very shallow shells. For shells deeper than that subsequent terms in the original expressions should also be included in the order of gradually increasing significance of each. Such a selective choice of terms in the governing equations can significantly reduce the computational load, increase the efficiency of analysis and still maintain the required level of accuracy.

\section{Conclusions}

In continuum mechanics, proponents of plate and shallow shell theory would seldom depart from the belief that the bending plays an important role in determining the load resistance mechanism, which is 
true in their realm when considering finite deformations. The present research has shed more light into the other extreme of deep shells, where it is found that bending plays very little or no part in the strength mechanism.

The intricacies of medium curvature range of shells have also proved the often experienced dilemma by the practicing engineer to be true. Within this range, which has wide practical applications, the problem has to be approached with extreme caution when formulating the theoretical as well as the numerical methods.

The mostly quantitative results presented here for the particular shell types could serve as a useful guideline for other types of shell problems too. It has to be remembered that the present results are strictly within the statically stable region and the same could be not applicable to dynamic phenomena, where the small nonlinearities at an initial stage might play havoc in the stability mechanism.

\section{References}

1) Flügge, W.: Tensor Analysis and Continuum Mechanics, Springer-Verlag, New York (1972).
2) W. Pietraszkiewicz: Geometrically Nonlinear Theories of Thin Elastic Shells, Advances in Mechanics, 12 (1989), pp. 51 130.

3) Shinoda, T. T. George, Fukuchi, N.: A Detailed Analysis of the Theory of Thin Shells and some Particular Applications, Trans. of the West Japan Society of Naval Architects, No. 80 (1990), pp. $171 \sim 193$.

4) Fukuchi, N., T. George, Shinoda, T.: Dynamic Instability Analysis of Thin Shell Structures subjected to Follower Forces, (2 nd Report) Numerical Solutions and some Theoretical Concepts, Journal of the Society of Naval Architects of Japan, No. 171 (1992), pp. 597 609.

5) T. George, Fukuchi, N.: Dynamic Instability Analysis of Thin Shell Structures subjected to Follower Forces, (6 th Report) Dynamic Threshold Characteristics and Post-critical Stability, Journal of the Society of Naval Architects of Japan, No. 176 (1994), pp. 319 330.

6) Fukuchi, N., T. George: Governing Equations for the Dynamic Stability of Thin Shells subjected to Follower Forces and some Numerical Results, Computational Mechanics' 95, Springer (1995), pp. 1541 1546. 